\title{
El dilema ético de la fluoración del agua potable
}

\author{
Carolina Mendoza V ${ }^{1 a}$ \\ The ethical dilemma \\ of water fluoridation
}

Dental caries remains a worldwide public health problem because of its magnitude and impact on affected people's quality of life. Among preventive strategies, water fluoridation is one of the most important, but its value still remains uncertain after more than a half of Century of its use. The aim of this study is to analyse some of the ethical arguments for and against water fluoridation and to determine if empirical data allow to decide if there are correct policies from a bioethical perspective. Autonomy, compulsory medication (mass medication), precautionary principle, justice in health care and ethics of protection are discussed. It is concluded that fluoridation is beneficial and that there is no ethical reason to oppose it, based on a specific kind of ethics developed to analyse and clarify complex public health's issues (Rev Méd Chile 2007; 135: 1487-93).

(Key words: Ethics; Fluoridation; Public Health)

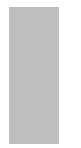

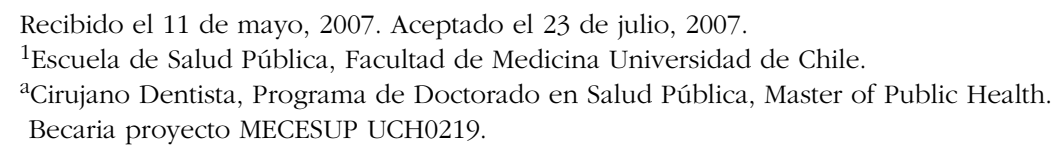

$\mathrm{L}$ a caries es una enfermedad crónica, infecciosa y transmisible que se caracteriza por la desmineralización de la superficie dentaria por la acción de los ácidos producidos por el metabolismo bacteriano de los carbohidratos simples de la dieta $^{1}$. Puede presentarse en cualquier etapa de la vida $y$, si no es tratada a tiempo, puede causar intenso dolor y la pérdida de los dientes afectados, lo que a su vez repercute en la capacidad masticatoria, fonética o estética. La Organización Mundial de la Salud plantea que la caries continúa siendo un importante problema de salud pública a nivel mundial, tanto por su magnitud, como por el impacto que provoca en la calidad de vida de

Correspondencia a: Carolina Mendoza V. Tel: 9786546. Fax: 7377121. E mail: caromendoza@med.uchile.cl

quienes la padecen. Se estima que en el año 2003 afectaba al $60-90 \%$ de los escolares y a la gran mayoría de los adultos de los países desarrollados, mientras que en los países de América Latina y Asia, la caries continúa siendo la enfermedad bucal más prevalente ${ }^{2,3}$. En Chile, la Encuesta Nacional de Salud (2003) estimó que cerca de dos tercios de la población mayor de 17 años sufre de caries $^{4}$, mientras que entre los escolares, la prevalencia alcanzaría al 85\%5. Esta situación ha sido reconocida por el país, siendo actualmente la salud bucal considerada como una de las prioridades de salud, tanto por sus características epidemiológicas como por la percepción e impacto de estas patologías en la salud general y calidad de vida de la población ${ }^{6}$.

Dadas las características de la caries y la situación epidemiológica descrita, las medidas 
preventivas han adquirido un rol fundamental como estrategias para enfrentar el problema. De entre ellas destaca la fluoración del agua potable, tal vez una de las más interesantes y controvertidas medidas de salud pública odontológica ${ }^{7}$. Su historia se remonta a principios del siglo XX y luego de más de un siglo de trayectoria, continúa siendo materia de intenso debate. Entre los aspectos más controvertidos están su carácter ético ${ }^{8}$ y sus potenciales efectos adversos en la salud, destacando por la plausibilidad biológica e impacto en la salud pública, el presunto riesgo de fracturas de cadera y cáncer ${ }^{9}$. El objetivo de este trabajo es analizar algunos de los argumentos a favor y en contra de la fluoración del agua potable que se discuten en la literatura científica, con el propósito de establecer si la información empírica permite definir el carácter ético de una medida de salud pública.

\section{FluOración DEL AGUA POTABLE}

Fue a partir de observaciones hechas por odontólogos estadounidenses y europeos a principio del siglo XX que se descubrió el efecto protector del fluoruro sobre la caries. La presencia del "esmalte moteado" en niños (dientes con pigmentaciones de color café) permitió revelar los efectos de los iones de fluoruros en los dientes. Los fluoruros se encuentran naturalmente en el agua, pero en las comunidades donde se observaba el "esmalte moteado", se encontraban en concentraciones inusualmente altas. Además de las pigmentaciones, condición que pasó a llamarse "fluorosis", estas comunidades presentaban menor prevalencia de caries, lo que motivó a los investigadores a buscar el nivel óptimo de fluoruros en el agua de consumo, para maximizar su efecto protector y minimizar el riesgo de fluorosis en la población. HT Dean ${ }^{10}$, de NIH Estados Unidos, planteó que ese nivel óptimo de fluoruros estaba alrededor de $1 \mathrm{ppm}(1 \mathrm{mg} / \mathrm{l})$, admitiendo pequeñas variaciones según la temperatura ambiental. La Asociación Dental Americana recomendó oficialmente la fluoración del agua potable para la prevención de caries en la población de Estados Unidos en la década de los 50, propuesta que fue apoyada posteriormente por la Organización Mundial de la Salud y la Organización Panamericana de la Salud, extendiéndose su aplicación a diversos países en el mundo ${ }^{1,3,10,11}$.

A pesar del impacto que generó en la salud pública y en la odontología el descubrimiento del rol de los fluoruros en la prevención de la caries, la fluoración del agua potable ha sido objeto de intensas discusiones éticas. Mientras algunos la celebran como uno de los diez principales logros de la Salud Pública en el siglo $\mathrm{XX}^{12}$, otros consideran que es una medida poco ética que viola principios bioéticos fundamentales.

\section{ARGumentos EN CONTRA DE LA MEDIDA}

Principialismo. Como medida de salud pública, la fluoración del agua potable pretende actuar sobre la comunidad como un todo, buscando protegerla de la ocurrencia de caries y por lo tanto, buscando el bien común. Uno de los primeros argumentos planteados en contra de la medida se relaciona con el principio de autonomía ${ }^{8}$. Cohen y Locker analizan la fluoración en términos de beneficencia y autonomía, estableciendo que la búsqueda del bien de los otros (principio de beneficencia) entra en conflicto con el principio de autonomía de los individuos, en la medida en que la fluoración del agua potable les coarta la libertad de elegir ${ }^{13}$. Sin embargo, el análisis bioético centrado en estos principios (beneficencia y autonomía) parece ser inapropiado para la reflexión en salud pública, ya que éstos están basados en la relación individual médico-paciente o investigador-probando ${ }^{14}$, siendo que en el caso de la salud pública, la relación está dada entre colectivos y por lo tanto es mucho más compleja. Más aún, el principio de autonomía resulta difícilmente aplicable en situaciones como ésta, en la cual es técnicamente imposible aplicar la medida sólo en aquellos que voluntariamente la aceptan, mientras que el principio de beneficencia resulta muy difícil de evaluar. Por lo tanto, la reflexión en base a la beneficencia y autonomía, tratando de establecer cuál de los principios debe predominar para así establecer el carácter ético de la fluoración del agua, no es adecuada para resolver el dilema, ya que no considera la complejidad ni la magnitud de las interrelaciones y problemas que se dan entre colectivos, siendo el conflicto que puede haber entre autonomía individual y bien común, ejemplo de aquello. 
Fluoración como medida de "medicación masiva". Otros argumentos en contra de la medida se refieren a la naturaleza de la intervención propiamente tal. Autores como Cross y Carton ${ }^{15}$ plantean la necesidad de reclasificarla para que sea considerada como una intervención de "investigación médica" y que por lo tanto se rija por la ética de la investigación. Para plantear esta propuesta, los autores asumen que los compuestos de fluoruros que se agregan al agua son medicamentos y que por lo tanto, su administración "experimental" debe regularse éticamente como cualquier investigación en seres humanos. Esto implica considerar a la comunidad como un grupo de probandos, quienes a través de un consentimiento informado deberían ser notificados individualmente sobre los riesgos y beneficios de la intervención, así como del carácter voluntario de su participación y de su derecho a abandonarla cuando lo consideren necesario, sin perjuicio alguno. Además, los autores plantean que la fluoración del agua potable constituye también un acto de medicación masiva de la comunidad, lo que violaría los derechos humanos fundamentales que establecen la obligación de proteger a los individuos de ser sometidos a intervenciones médicas sin su consentimiento ${ }^{15}$.

Sin embargo, para considerar la fluoración como una investigación, es necesario explicitar la pregunta a la cual se quiere dar respuesta a través del experimento. En este caso, no hay una pregunta a responder, ya que la fluoración es una medida preventiva de salud pública de efectividad comprobada $^{16}$ que se aplica con el propósito de disminuir la incidencia de caries en una población. Luego, la fluoración no puede ser considerada como una intervención de investigación médica. Pero además, los autores en su análisis no consideran la caries como un problema de salud pública. Todo lo contrario, plantean que al no constituir una amenaza directa a la vida, las personas podrían incluso prescindir de tratamiento. Claramente este planteamiento no se ajusta a la realidad de las comunidades con alta prevalencia de caries, las que ven perjudicada no sólo su salud bucal y general, sino que también su calidad de vida ${ }^{17}$. La fluoración del agua potable busca reducir los niveles de caries en la población, lo que implica que cuando la caries no constituye un problema de salud pública, la aplicación de la medida pierde todo sentido. Luego, no correspon- de discutir sobre su aplicación a menos que haya justificación técnica para realizarla.

Principio de precaución. Otra forma de analizar la fluoración del agua potable es señalando que los compuestos fluorados que se incorporan para corregir la concentración son sustancias químicas con riesgos desconocidos que se agregan al agua de consumo humano. Esta postura incluye cuestionamientos sobre la composición y el origen de los compuestos fluorados utilizados, planteando que al no estar aprobados por las instituciones que velan por la seguridad de los alimentos y drogas de consumo humano, su inocuidad para la salud no estaría asegurada ${ }^{15}$. Además, existe preocupación por las dosis utilizadas y la poca estabilidad de las concentraciones en el agua a lo largo del tiempo, ya que hay evidencia de efectos tóxicos de altas dosis de fluoruros en el tejido cerebral y reproductor de $\operatorname{ratas}^{18}$. Sin embargo, con el uso de dosis cercanas a $1 \mathrm{ppm}$ no hay evidencia de efectos tóxicos en humanos, siendo el único efecto adverso comprobado la presencia de fluorosis dental ${ }^{16}$, la que para quienes rechazan la fluoración del agua no sólo corresponde a un biomarcador de la ingesta de fluoruros ${ }^{18}$, sino que es la primera manifestación visible de intoxicación por fluoruros ${ }^{19}$.

Estos potenciales efectos tóxicos de los fluoruros para la salud justificarían la suspensión de la medida bajo el "principio de precaución", el que plantea la necesidad de adoptar una actitud de cautela frente a la incertidumbre en torno a los posibles riesgos de una intervención ${ }^{20}$. Bajo este argumento, se plantea que la falta de evidencia en torno a los riesgos y beneficios de la fluoración del agua potable hacen que el apoyo a esta medida sea considerado inmoral ${ }^{13}$. Si embargo, si bien no existen regulaciones sobre la naturaleza de los compuestos fluorados a utilizar, sí existe control sobre las dosis máximas de fluoruros permitidas. En el caso de Chile, la concentración máxima permitida es de $1,5 \mathrm{mg} / \mathrm{l}$, existiendo también normas sobre las variaciones diarias, mensuales y anuales máximas permitidas ${ }^{21}$.

En cuanto a los presuntos efectos adversos de la fluoración del agua, se han evaluado las posibles asociaciones de los fluoruros con fracturas óseas, problemas de tiroides, de fertilidad y renales entre otros, sin lograr acuerdo ${ }^{18,22-25}$ ni resultados con- 
sistentes. El esfuerzo más sistemático realizado corresponde a la revisión de McDonagh y $\mathrm{col}^{16}$, en la cual se evaluaron todos los estudios existentes sobre la efectividad y seguridad de la fluoración del agua. Sus resultados indican que la fluoración a dosis de $1 \mathrm{ppm}$ reduce la incidencia de caries en los niños, siendo la presencia de fluorosis dental el único efecto adverso demostrado ${ }^{16}$. Más aún, las evaluaciones del impacto ambiental de la fluoración del agua revelan que no hay evidencia de daño, siendo considerada una medida ambientalmente segura ${ }^{23}$.

Por lo tanto, parece haber acuerdo en los posibles efectos tóxicos de altas concentraciones de fluoruros, de ahí las normas sobre concentración y variaciones máximas permitidas. Sin embargo, a concentraciones cercanas a 1 ppm, la fluorosis dental es el único riesgo conocido y su impacto en la salud se reduce a consideraciones estéticas $^{16}$. Esto implica que los riesgos son conocidos, cuantificables y controlables, por lo que no se justifica suspender la medida. Más aún, la precaución permite el uso de agentes con riesgos conocidos y controlables, con el compromiso de seguir indagando riesgos potenciales, considerando idealmente la opinión de la comuni$\mathrm{dad}^{20}$

\section{Argumentos A FAVOR DE LA MEDIDA}

Los argumentos éticos a favor parten de la base que la fluoración corresponde a una medida de "fortificación de alimentos", entendiendo que se trata del ajuste controlado de la concentración de fluoruros hasta alcanzar niveles óptimos para disminuir la incidencia de caries. Dado que los fluoruros se encuentran naturalmente en el agua, el ajuste de su concentración para mantener la salud dental no difiere de medidas ampliamente aceptadas ${ }^{26,27}$. Es el caso de la fortificación de la harina con ácido fólico para prevenir los defectos congénitos del tubo neural, medida en vigencia en Chile desde el año $2000^{28,29}$. Luego, la fluoración no implica adicionar compuestos extraños al agua, sino que sólo ajustar la concentración de compuestos naturalmente presentes (fluoruros) para lograr el máximo beneficio (prevención de caries) con el menor riesgo posible (fluorosis dental)

\section{JUSTICIA SANITARIA}

Aceptando lo anterior, uno de los argumentos más importantes a favor de la fluoración del agua potable tiene relación con la justicia sanitaria. La reflexión ética sobre justicia se centra en la justa distribución de bienes y servicios, siendo el contexto socioeconómico un factor importante a considerar. Comprende la reflexión sobre la manera de distribuir equitativamente los recursos siempre escasos, considerando aspectos relacionados con la cobertura de necesidades, apoyo a las acciones sanitarias más efectivas y distribución orientada hacia la disminución de desigualdades en salud ${ }^{30}$

Cobertura de necesidades. Una de las principales características de la fluoración del agua es su universalidad, en la medida que beneficia a toda la comunidad abastecida por agua potable, suministrándole niveles óptimos de fluoruros. Si se considera que toda la población es susceptible de padecer caries a lo largo de toda su vida, resulta lógico pensar que el beneficio debería extenderse a toda la población. En el caso de Chile, más del $70 \%$ de la población recibe agua potable fluora$\mathrm{da}^{5}$, cobertura difícil de alcanzar con intervenciones que impliquen otras vías de administración de fluoruros. Por ejemplo, dado que la cantidad de horas semanales de atención odontológica en el Sistema Nacional de Servicios de Salud es inferior al estándar definido para dar atención básica a los grupos beneficiarios ${ }^{31}$, es esperable que la aplicación tópica de productos fluorados (barnices y geles) que requieren la participación directa de odontólogos alcancen una cobertura muy deficiente. Por otro lado, los programas de fluoración de leche impulsados por la Junta Nacional de Auxilio Escolar y Becas limitan su cobertura a la población infantil beneficiaria del Programa de Alimentación Escolar ${ }^{32}$. Luego, la fluoración del agua potable es la única medida capaz de dar cobertura a toda la población en riesgo.

Costo/efectividad de la medida. Otro aspecto a considerar en la reflexión sobre justicia es la efectividad de la medida y sus costos económicos asociados. Se ha estimado que el costo por persona de la fluoración del agua durante toda la vida es menor que el costo de una restauración 
dental $^{25}$, mientras que otras estimaciones del costo de la fluoración hablan de USD 0.03 per capita/año en Sao Paulo $^{8}$ y USD 0.72 per capita/ año en EE.UU. de $\mathrm{NA}^{3}$. Por otro lado, mientras mayor sea la cobertura de la red de agua potable a fluorar, menor será el costo de implementación y mantención a absorber por cada miembro de la comunidad, aunque tanto en comunidades pequeñas como grandes se logra un ahorro por los tratamientos dentales evitados ${ }^{33}$. La fluoración del agua potable continúa siendo la medida más costo efectiva de implementar y, si consideramos que los recursos son escasos, resulta éticamente correcto invertirlos de la manera más eficiente posible.

Disminución de las desigualdades. El tercer elemento a considerar cuando se habla de justicia se refiere a la capacidad de la medida para disminuir las desigualdades. La fluoración del agua potable no sólo ha demostrado ser capaz de reducir la presencia de caries en la población ${ }^{16}$, sino que además ha logrado disminuir las desigualdades de salud bucal generadas por las diferencias socioeconómicas. En las zonas fluoradas existe menor prevalencia de caries en la población, pero además las desigualdades parecen ser menos intensas Esto se debe a que las personas de menores recursos tienen menos acceso a otras fuentes de fluoruros, como son las pastas dentales o las aplicaciones realizadas por dentistas. Luego, el agua fluorada se transforma en la única fuente de fluoruros y quienes carecen de agua fluorada, simplemente se ven privados del beneficio preventivo de concentraciones óptimas de fluoru$\operatorname{ros}^{34}$.

De lo anterior se concluye que la fluoración del agua potable es una medida justa, ya que abarca a toda la población en riesgo, es la medida más costo efectiva y tiene la capacidad de reducir las desigualdades de salud bucal generadas por las diferencias socioeconómicas, elementos que constituyen los requisitos de la justicia sanitaria.

\section{ETICA DE LA PROTECCIÓN}

Si bien la fluoración del agua potable resulta ser una medida justa según el análisis anterior, la complejidad de los problemas e intervenciones de salud pública obligan a reflexionar sobre aspectos que van más allá de la justicia. En esa dirección se plantea la ética de la protección, la que constituye una propuesta para el análisis ético de los dilemas en salud pública, así como una estrategia de investigación. Está basada en cinco condiciones, abordando de manera directa la justificación de las restricciones de la autonomía impuesta por algunas intervenciones de salud pública. Estas condiciones son: 1) la intervención debe abordar un problema de salud pública sentido como importante por la población; 2) la intervención debe demostrar ser la mejor estrategia existente capaz de disminuir el daño o mantener el bienestar; 3) la intervención debe ser la más eficaz (magnitud del daño que resuelve) y efectiva (relación beneficios-costos) de las alternativas existentes; 4) los riegos que implica la intervención deben ser aleatorios; es decir, no han de afectar desproporcionadamente a un grupo específico; 5) si la medida cumple todos estos requisitos planteados por la ética de la protección, será considerada legítima y se deberá asegurar su implementación con la máxima efectividad, justificándose su obligatoriedad. Por lo tanto, bajo estas condiciones es éticamente aceptable limitar la autonomía individual para asegurar el buen resultado de la medida de salud pública ${ }^{20}$.

$\mathrm{Al}$ aplicar el análisis propuesto por la ética de protección, la fluoración del agua resulta ser una medida éticamente adecuada. Responde a un problema importante de salud pública, como es la caries dental; ha demostrado ser efectiva en disminuir la prevalencia de caries, lo que implica que protege la salud bucal; es la más costo efectiva de las alternativas; y los riesgos son conocidos, sustentables y aleatorios. Luego, la fluoración del agua potable es una medida legítima que debe ser apoyada, justificándose la limitación de la autonomía individual en nombre del bien común.

\section{Conclusiones}

El análisis realizado desde la perspectiva bioética sobre la fluoración del agua potable constituye un ejemplo de la reflexión ética necesaria en salud pública. Esta reflexión permite concluir que, al abordar problemas colectivos, las medidas de 
salud pública deben respetar la equidad de acceso y asegurar la obtención de beneficios para la sociedad, restringiendo en cierto grado la autonomía individual, si fuera necesario. Por otra parte, queda en evidencia la necesidad de discutir los aspectos éticos de una medida de salud pública,

\section{REFERENCIAS}

1. Fluoridation of drinking water to prevent dental caries. (Cover story). MMWR: Morbidity \& Mortality Weekly Report 1999; 48: 933-40.

2. Petersen Pe, Bourgeois D, Ogaiwa H, Estupinan-Day $S$, NDiaYe C. The global burden of oral diseases and risks to oral health. Bull World Health Organ 2005; 83: 661-9.

3. Jones S, Burt BA, Petersen Pe, Lennon Ma. The effective use of fluorides in public health. Bull World Health Organ 2005; 83: 670-6.

4. MINSAL. Encuesta Nacional de Salud Chile 2003. Disponible en: http://epi.minsal.cl/epi/html/invest/ENS/ENS_mayo2004.pdf [Consultado en mayo 2007].

5. MINSAL. Diagnóstico de Situación de Salud Bucal Disponible en: http://www.minsal.cl/ici/ salud_bucal/documentos/PerfilEpidemiologico.pdf [Consultado en mayo 2007].

6. MINSAL. Lineamientos estratégicos salud bucodental 2000-2010. Disponible en: http:// www.minsal.cl/ici/salud_bucal/documentos/ lineamientos_estrategicos_2000_2010.pdf [Consultado en mayo 2007].

7. Colquhoun J. Why I changed my mind about water fluoridation. Perspect Biol Med 1997; 41: 2944.

8. Kalamatianos PA, Narvai PC. Ethical aspects of the use of fluoride products in Brazil: a view of public health policy formulators. Ciênc. saúde coletiva 2006; 11: 63-9.

9. Medical-Research-Council. Water fluoridation and health 2002. Disponible en: http://www.mrc.ac.uk/Utilities/Documentrecord/index.htm?d=MRC002482 [Consultado en mayo 2007].

10. Maier F. Historia y desarrollo. En: OPS/OMS, ed. Fluoruración del agua potable. Washington, DC: 1971; 11-23.

11. NARVAI PC. Dental caries and fluorine: a twentieth century relation. Ciênc. saúde coletiva 2000; 5 : 381-92. debido principalmente a la falta de buenos instrumentos de ponderación empírica. Es por esto que se hace necesario desarrollar una ética propia de la salud pública, siendo la ética de protección una propuesta que considera el carácter colectivo de las medidas de salud pública.

12. Ten great public health achievements - United States, 1900-1999. MMWR: Morbidity \& Mortality Weekly Report 1999; 48: 241-3.

13. COHEN H, Locker D. The science and ethics of water fluoridation. J Can Dent Assoc 2001; 67: 578-80.

14. Schramm FR, Kottow M. Principios bioéticos en salud pública: limitaciones y propuestas. Cad Saude Publica 2001; 17: 949-56.

15. Cross DW, Carton RJ. Fluoridation: a violation of medical ethics and human rights. Int J Occup Environ Health 2003; 9: 24-9.

16. McDonagh MS, Whiting PF, Wilson PM, Sutton AJ, Chestnutt I, Cooper J, et al. Systematic review of water fluoridation. BMJ 2000; 321: 855-9.

17. Petersen Pe, Bourgeois D, Ogawa H, Estupinan-Day S, NDiaye C. The global burden of oral diseases and risks to oral health. Bull World Health Organ 2005; 83: 661-9.

18. Connett P. Water fluoridation a public health hazard. Int J Occup Environ Health 2006; 12: 8891.

19. Gibson SL, Gibson RG. Water fluoridation. Clearer evidence of benefits and risks is needed. BMJ 2001; 322: 1487-8.

20. Koтtow M. Bioética y salud pública. En: Kottow M, ed. Bioética en salud pública. Santiago: Editorial Puerto de Palos, 2005; 143-71.

21. MINSAL. Normas de uso de fluoruros en la prevención odontológica 1998. Disponible en: http://www.minsal.cl/ici/salud_bucal/ documentos\%5Cnormas_de_uso_fluoruros_en_la_prevencion_odontologica.pdf [Consultado en mayo 2007].

22. ConnetT P. Scientific evidence fails to support fluoridation of public water supplies. Int J Occup Environ Health 2005; 11: 215-6.

23. Polıick H. Water Fluoridation and the environment: current perspective in the United States. Int J Occup Environ Health 2004; 10: 343-50.

24. PolLICK HF. Concerns about water fluoridation, IQ, and osteosarcoma lack credible evidence. Int J Occup Environ Health 2006; 12: 91-4. 
25. Poluick H. Scientific evidence continues to support fluoridation of public water supplies. Int J Occup Environ Health 2005; 11: 322-6.

26. British-Fluoridation-Society. The ethics of fluoridation Disponible en: http://www.bfsweb.org/ ethicsoffluorida.html [Consultado en mayo 2007].

27. Avebury L. Fluoridation and individual freedom. Br Dent J 1984; 156: 277.

28. Corral SE, Moreno SR, Pérez GG, Ojeda BM, Valenzuela GH, Reascos MM, et al. Defectos congénitos cráneo-encefálicos: variedades y respuesta a la fortificacion de la harina con acido folico. Rev Méd Chil 2006; 134: 1129-34.

29. Hertrampf E, Cortes F. Folic acid fortification of wheat flour: Chile. Nutr Rev 2004; 62: 44-9.

30. Kotтow M. Teorías de justicia. En: Kottow M, ed.
Bioética en salud pública. Santiago: Editorial Puerto de Palos, 2005; 173-87.

31. MINSAL. Catastro nacional de recursos humanos odontológicos 2003. Disponible en: http:// www.minsal.cl/ici/S_ $1 /$ saludbucal/ Catastro\%20RRHH\%20a\%20color.pdf [Consultado en mayo 2007].

32. JUNAEB. Junaeb al día 2005. Disponible en: http://www.junaeb.cl/noticias/doc/ boletin_agosto.pdf [Consultado en mayo 2007].

33. Peres Ma, Fernandes SL, Glazer Peres K. Inequality of water fluoridation in Southern Brazil-the inverse equity hypothesis revisited. Soc Sci Med 2004; 58: 1181-9.

34. Burt BA. Fluoridation and social equity. J Public Health Dent 2002; 62: 195-200. 